EPJ Web of Conferences 58, 02011 (2013)

DOI: $10.1051 /$ epjconf/20135802011

(C) Owned by the authors, published by EDP Sciences, 2013

\title{
Peering into the Past
}

\section{Cosmology as a Tool for Constraining GR}

\author{
Stefano Camera ${ }^{1, a}$ \\ ${ }^{1}$ CENTRA, Instituto Superior Técnico, Universidade Técnica de Lisboa, Av. Rovisco Pais 1, 1049-001 \\ Lisboa, Portugal
}

\begin{abstract}
In the last few decades, cosmological measurements have reached an unprecedented level of precision, and cosmology is now on the threshold of a new era of observational evidence. In the next future, a vast number of telescopes, satellites and surveys will provide us with accurate, multi-wavelength data that will allow us to investigate the fundaments of our theoretical model of the Universe. Here, we depict, with some useful example, how such a gaze to the deepest cosmos could help in better understanding the laws of gravity and perhaps detecting departures from GR. Specifically, we show recent constraints on well-known alternative gravity theories from some of these next-generation instruments.
\end{abstract}

\section{Introduction}

In the last decades, cosmologists proposed several models alternative to the concordance $\Lambda$ cold dark matter $(\Lambda \mathrm{CDM})$ paradigm. These models attempt to find an agreement at least as good as that of $\Lambda \mathrm{CDM}$ with current cosmological datasets, viz. the temperature anisotropy pattern of the cosmic microwave background (CMB) radiation [1], the dynamics of the large-scale structure of the Universe [2] and the present-day cosmic accelerated expansion [3]. However, in these theories, crucial topics such as the missing mass in galaxies and galaxy clusters and the current Universe's accelerated expansion are not explained by usual cold dark matter and the cosmological constant $\Lambda$. On the contrary, these models rely on either the introduction of additional scalar or vector fields in the Universe's content or a modification of the law of gravity. For a detailed description of these hypotheses, as well as of their impact on cosmology, we suggest Refs $[4,5]$.

To the aim of achieving a better understanding of the Universe and of refining our current cosmological model-particularly, those aspects related to the so-called "dark sector"—a strong effort has been put by the community in the developing of a new generation of telescopes, satellites, experiments and surveys. On of their most interesting and promising aspects, is the vast variety of observables, which will widely span the electro-magnetic spectrum. To just give some name, leaving a deeper search to the interested reader, we may quote: the ongoing CMB experiment Planck, in the microwave band; LOFAR and the SKA pathfinders such as EMU and WODAN, in the radio band; SDSS and the CFHT Survey Legacy, DES, LSST, Pan-STARRS, and the future Euclid satellite, for the optical band.

ae-mail: stefano.camera@ ist.utl.pt

This is an Open Access article distributed under the terms of the Creative Commons Attribution License 2.0, which permits unrestricted use, distribution, and reproduction in any medium, provided the original work is properly cited. 
Here, we are interested in the potential of these next generations of cosmological experiments in scrutinising and testing alternative and modified version of the theory of gravitation-that is to say, Einstein's General Relavity (GR). Therefore, there is one main question we have to ask ourselves: "Why should cosmology be good as a tool for testing GR?" And the answer is that, after radiation/matter decoupling, gravity has basically been the sole and major force leading the evolution of the Universe and the formation of the large-scale structure we see-and, most of all, we can measure-today. The understanding of the fundamental laws behind the formation of these structures is therefore a promising and powerful tool we have at our disposal.

The structure of this paper will be threefold: in $\S 2$, we shall describe the rôle of GR in the $\Lambda$ CDM model, and why the idea of modifying GR has arisen; secondly, $\S 3$ will outline the reasons why deep and large-scale cosmology is one of the best possible arenas for testing GR and its competitors, and we shall as well introduce some cardinal cosmological observables; eventually, some conclusions will be drawn in $\S 4$, by also showing recent constraints and forecasts on some well-known modified gravity models.

\section{The Concordance Cosmological Model}

The $\Lambda$ CDM model is based on two cornerstones: on the one hand, GR encodes the properties of spacetime; on the other hand, the Standard Model of Particle Physics provides us with the energy and matter species that fill the cosmos. The interaction of the two theories describes the geometry of the Universe and its evolution in time.

As mentioned above, the tiny anisotropies in the almost-uniform CMB, the unexpected faintness of distant Type Ia supernovæ and the hint of a "cosmic web" emerging from observations of the largescale structure of galaxies and galaxy clusters are three strong experimental pieces of evidence in favour of the $\Lambda \mathrm{CDM}$ model. However, all of this proposes the picture of a present-day spatially-flat Universe where only $\sim 4 \%$ of the total energy budget is accounted for by standard baryonic matter, besides a negligible amount of energy due to photons and neutrinos. The remaining $\sim 85 \%$ of the nonrelativistic matter in the Universe is then constituted by dark matter, whilst the cosmological constant is responsible for the great majority of the total energy content of the cosmos, more than $70 \%$ of the total.

From structure formation, we know that the $\Lambda$-like component can not clump on the scales covered by the galaxy surveys and below. Thus, it must be something different from standard matter and radiation and even from dark matter. Although the cosmological constant is a perfectly viable description for all observational evidence we have, it is deeply unsatisfactory from the viewpoint of theoretical physics. This is because there is an enormous disagreement between the observed value of $\Lambda$ and its theoretical estimate [6].

To find possible solutions to this "cosmological constant problem", many routes have been tested, particularly in the very last decades. Here, we are interested in studying those ones which involve a modification of the law of gravity. Indeed, the cosmological constant was originally proposed as a geometric term, i.e. a constant term in the left-hand (geometric) side of Einstein's equation-thus adding no extra component to the stress-energy tensor. By generalising this approach, one can argue whether it is possible to reproduce the current cosmic accelerated expansion by adding a non-constant time-dependent term in Einstein's tensor. The effort of modifying the action of gravity actually dates back to just few years after Einstein's seminal papers. Since then, a vast plethora of theories has been proposed. For the purposes of this introductory work, we shall focus on one of the most developed and studied approach, which is also amongst the most radical modifications to GR, i.e. modified-action theories [7, e.g.]. In modified-action theories directly modify the law of gravity by generalising the Hilbert-Einstein Lagrangian. 


\section{Deep and Large-Scale Cosmology}

As we have mentioned, type Ia supernovæ, high-redshift gamma-ray bursts and distance indicators from the CMB put strict constraints on the Universe's background evolution, which appears to be in good agreement with the $\Lambda \mathrm{CDM}$ model. As a consequence, any modified-gravity model which attempts to give a plausible alternative explanation for the late-time cosmic accelerated expansion must be degenerate with $\Lambda \mathrm{CDM}$ at background level. In other words, all viable cosmological models should share the same Hubble parameter (at least in the matter-dominated epoch), which reads

$$
H(z)=H_{0} \sqrt{\Omega_{m}(1+z)^{3}+\Omega_{\Lambda}},
$$

with $H_{0}$ the Hubble constant, $\Omega_{m}$ the total matter density in units of the critical density, $\Omega_{\Lambda}$ the cosmological-constant contribution, which equals $1-\Omega_{m}$ in a flat Universe, and $z$ the redshift.

On the other hand, different gravity models do behave differently when we switch on cosmological perturbations. Indeed, GR predicts a standard Poisson's relation between the large-scale Newtonian potential $\Phi$ and the matter-density fluctuations $\delta$, namely

$$
\nabla^{2} \Phi=-\frac{3}{2} H_{0}^{2} \Omega_{m}(1+z) \delta
$$

furthermore, dust has no anisotropic stress, that is to say that the two Bardeen's potentials $\Phi$ and $\Psi$ are equal in modulus. Contrarily to this, modified gravity theories usually introduce a time- and scaledependent modification to the Newtonian gravitational constant, $\mu(k, z)$, which enters the right-hand side of Eq. (2), as well as a non-trivial relation $\Phi=\eta(k, z) \Psi$. We want to emphasise that this is a general though approximate approach to modified gravity, but nonetheless a good exercise for the purposes of this piece of work, whose underlying idea is to show how cosmological observables capable of probing perturbed quantities such as $\delta, \Phi$ and $\Psi$ may discriminate between GR and competing theories. Hereafter, we shall introduce some of those large-scale observables.

\subsection{The Growth Factor}

Let us start with the growth factor. Indeed, the primary effect of modified gravity will be on the growth of structure. The time evolution of the density field can be a sensitive probe of not only the expansion rate of the Universe but also its matter content. In a flat, matter-dominated Universe we have that $\delta \propto a$, where $a$ is the scale factor. We can parameterise deviations from this behaviour in terms of the growth factor

$$
f(z)=\left.\frac{\mathrm{d} \ln \delta}{\mathrm{d} \ln a}\right|_{a=1 /(1+z)} .
$$

It is worth stressing that the linear density contrast $\delta(a)$ used in the equation above actually holds only in the subhorizon limit, i.e. under the approximation $k \gg \mathcal{H}, \mathcal{H}^{\prime} / \mathcal{H}, \mathcal{H}^{2}$, with $\mathcal{H}=a H$ the expansion rate in terms of the conformal time. However, for a preliminary investigation, we may still use Eq. (3), which is well suited for galactic and galaxy-cluster scales.

\subsection{The 3D Matter Power Spectrum}

Another important observable, beyond the growth function, is the matter power spectrum. It encodes the information on how the density fluctuations cluster on different physical scales $k$. It is the Fourier transform of the two-point correlation function of $\delta(\mathbf{x})$ in real space, and at a given redshift $z$ it is defined via $\left\langle\delta_{\mathbf{k}}(z) \delta_{-\mathbf{k}^{\prime}}(z)\right\rangle=(2 \pi)^{3} \delta_{D}\left(\mathbf{k}-\mathbf{k}^{\prime}\right) P^{\delta}(k, z)$, where $\delta(\mathbf{x})$ and $\delta_{\mathbf{k}}$ form a Fourier pair, $\delta_{D}$ denotes 
Dirac's delta function, and $k=|\mathbf{k}|$. The shape of the power spectrum contains a wealth of information: the amplitude of clustering as a function of scale, its redshift dependence, how its shape on small scales is distorted by small scale velocities (known as redshift space distortions), and acoustic features imprinted by the baryons from pre-recombination (known as baryon acoustic oscillations) can all be used as distance indicators. The power spectrum can be estimated from surveys of galaxies or clusters of galaxies, the clustering properties of which can be directly related to the amplitude of fluctuations in the density field (under certain assumptions of how galaxies, or clusters, trace the density field, i.e. what is their bias).

\subsection{The Cosmic Shear Angular Power Spectrum}

Finally, we introduce cosmic shear, a first-order weak gravitational lensing effect. GR predicts that light beam paths are curved by the presence of matter. In the weak lensing framework, the deflection of light is small and we can thus use the Born approximation, where lensing effects are evaluated on the null-geodesic of the unperturbed (unlensed) photon [as exhaustive reviews, see 8,9]. All weak lensing observables may be expressed in terms of a convergence $\kappa$ and a (complex) shear $\gamma$ of the background image. Cosmic shear is one of the most useful manifestations of gravitational lensing. It is the alignment of nearby images on the sky due to intervening matter along the line of sight. Detection of dark matter on large scales through such cosmic shear measurements- the small, coherent distortion of distant galaxy images due to the large-scale distribution of matter in the cosmos-has recently been shown to be feasible. Since it is an integrated effect due to the large-scale distribution of matter along the line-of-sight direction, it is actually a $2 \mathrm{D}$, projected observable. Specifically, the cosmic-shear angular power spectrum $C^{\gamma}(\ell)$, where $\ell$ is the angular wave-number, is an integral over redshift of the gradient on the sky of the deflecting potential $\Upsilon \equiv(\Phi-\Psi) / 2$, which sources the lensing distortions. Such integral is weighted by a kernel $W(z)$ dependent on the Universe's geometry (through the radial comoving distance $\chi$ ) and on a selection function which describes the redshift distribution of the source images. Specifically,

$$
C^{\gamma}(\ell)=\frac{\ell^{4}}{4} \int \frac{\mathrm{d} z}{H(z)} \frac{W^{2}(z)}{\chi^{2}(z)} P^{\Upsilon}\left[\frac{\ell}{\chi(z)}, z\right],
$$

where $P^{\Upsilon}$ is the deflecting potential power spectrum, and Limber's approximation sets $k=\ell / \chi[10]$.

\section{Discussion and Conclusions}

After having outlined why and how cosmology is well suited for testing and constraining alternative theories of gravity, we now show some useful example [for a thorough treatment, see e.g. 5, 11].

First, we start with $f(R)$ gravity (probably the most investigated approach), where Ricci's scalar $R$ in the gravitational Lagrangian is replaced by a generic function $f(R)$. Recently, two models carefully designed to pass the local gravity tests but still providing an accelerated cosmic expansion have been proposed [12-14]. By the use of cosmic shear, it has been shown that the extra- $\Lambda \mathrm{CDM}$ parameters of these models can be constrained with a high degree of accuracy by next-generation weak-lensing experiments such as Euclid [15]. Specifically, the authors have found that the ratio $\sigma\left(\vartheta_{\alpha}\right) / \vartheta_{\alpha}$ between the errors on the model parameters and their fiducial values are within $0.1 \%$ and $0.01 \%$, when redshift tomography is fully exploited. Moreover, this can be further improved by using weak lensing as a tool for peak count [16, for more details].

Secondly, we move to so-called $f(T)$ theories [e.g. 17]. Looking for a correction to GR, it is instructive to remember that an equivalent formulation is represented by teleparallelism. In this theory, torsion, instead of curvature, is responsible for the gravitational interaction. In this scenario, 
gravitational interaction is not replaced by geometry, and the torsion acts as a force, allowing the interpretation of gravity as a gauge theory of the translation group. In much the same way as for $f(R)$ theories, one can obtain a generalised teleparallel gravity replacing the torsion $T$ with a generic function $f(T)$, thus opening the way to a rich phenomenology. In Ref. [18], the authors have illustrated that two proposed $f(T)$ models [19] turn out to be in very good agreement with a large combination of geometrical datasets. Nonetheless, if we look at the growth of perturbations, the two models - almost degenerate at background level—now present reduced $\widetilde{\chi}^{2}$ 's of 15.3 and 2.1, when fitted against $f(z)$ data.

Hence, we can conclude that cosmology is a really powerful instrument to the aim of testing GR, and that the understanding of the régime of cosmological perturbations will help us in a deeper comprehension of any possible deviation from Einstein's theory of gravitation.

\section{Acknowledgments}

SC thanks his collaborators for letting him show results from common projects. SC acknowledges support from FCT-Portugal under grant PTDC/FIS/100170/2008. SC's work is funded by FCT-Portugal under Post-Doctoral Grant SFRH/BPD/80274/2011. SC also gratefully thanks the organisers and Politecnico di Torino for inviting him to attend to The Time Machine Factory. Eventually, SC is indebted to Antonaldo Diaferio and Emanele Biolati for useful discussions.

\section{References}

[1] E. Komatsu et al. (WMAP), Astrophys. J. Suppl. 180, 330 (2009), 0803.0547

[2] A.G. Riess et al., Astrophys. J. 659, 98 (2007), astro-ph/0611572

[3] D. Larson, J. Dunkley, G. Hinshaw, E. Komatsu, M. Nolta et al., Astrophys. J. Suppl. 192, 16 (2011), 1001.4635

[4] L. Amendola, S. Tsujikawa, Dark Energy: Theory and Observations (Cambridge University Press, Cambridge, UK, 2010)

[5] T. Clifton, P.G. Ferreira, A. Padilla, C. Skordis, Phys. Rept. 513, 1 (2012), 1106.2476

[6] S. Weinberg, Rev.Mod.Phys. 61, 1 (1989), morris Loeb Lectures in Physics, Harvard University, May 2, 3, 5, and 10, 1988

[7] A. De Felice, S. Tsujikawa, Living Rev. Rel. 13, 3 (2010), 1002.4928

[8] M. Bartelmann, P. Schneider, Phys. Rept. 340, 291 (2001), astro-ph/9912508

[9] M. Bartelmann, Class. Quant. Grav. 27, 233001 (2010), arXiv:1010.3829

[10] N. Kaiser, Astrophys. J. 388, 272 (1992)

[11] L. Amendola et al. (Euclid Theory Working Group) (2012), arXiv:1206.1225

[12] A.A. Starobinsky, JETP Lett. 86, 157 (2007), arXiv:0706.2041

[13] W. Hu, I. Sawicki, Phys. Rev. D76, 064004 (2007), arXiv:0705.1158

[14] V.F. Cardone, S. Camera, A. Diaferio, JCAP 1202, 030 (2012), arXiv:1201.3272

[15] S. Camera, A. Diaferio, V.F. Cardone, JCAP 1107, 016 (2011), arXiv:1104.2740

[16] V. Cardone, S. Camera, R. Mainini, A. Romano, A. Diaferio et al. (2012), arXiv:1204.3148

[17] H.I. Arcos, J.G. Pereira, Int. J. Mod. Phys. D13, 2193 (2004), arXiv:gr-qc/0501017

[18] V.F. Cardone, N. Radicella, S. Camera, Phys. Rev. D85, 124007 (2012), arXiv:1204.5294

[19] P. Wu, H.W. Yu, Eur. Phys. J. C71, 1552 (2011), arXiv:1008.3669 
